\title{
A New Comprehensive Evaluation Method for Construction Results of the Power System Communication Networks
}

\author{
Biyao Huang \& Guojun Liu \& Huixia Ding \\ China Electric Power Research Institute, Beijing, China
}

Yunpo Wang

Beijing University of Posts and Telecommunications, Beijing, China

Qi Yang

State Grid Corporation of China, Beijing, China

Ying Zhang

State Grid Sichuan Information\&Communication Company, Sichuan, China

\begin{abstract}
Power system communication networks are responsible for data transmitting among different entities in power systems. This paper aims to propose a method for the comprehensive evaluation for construction results of the power system communication networks. Firstly, factors in a multi-criteria decision making approach can be arranged in a hierarchic structure based on the Analytic Hierarchy Process (AHP),then the Fuzzy Decision Theory (FDT) is used to combine the factors into one. The method proposed in this paper can be used to provide necessary basis for decision making with certain credibility. In the end, this paper provide a case for validating the method based on statistical data from State Grid Corporation of China.
\end{abstract}

KEYWORD: Power System Communication Networks; Comprehensive evaluation; Analytic Hierarchy Process; Fuzzy Decision Theory

\section{GENERAL INSTRUCTION}

The communication channel, which is used to provide information transmitting in remote monitoring, control and protection, plays an important role in the development of smart grid. Moreover, office automation and management information of the power enterprises are depending more and more on the broad-band and high speed communication network [1]. We can see that, electric power communication network carries the major business, and provides important guarantee to the security and stability of the grid operation and enterprise management.

As the world largest electric power communication network has been built in China, more sophisticatedly professional management is needed. For example, reasonable and scientific evaluation for construction results of the power system communication network can quantify the planning, construction and management of the power system communication network, and provide necessary basis for decision making with certain credibility [2].

Our motivation of the present work lies in building the comprehensive evaluation for construction results of the power system communication networks with the top-down analysis, which has not been studied yet. Based on the Analytic Hierarchy Process (AHP) [3] and Fuzzy Decision Theory (FDT) [4-5], the structure of this paper will be as follows. In section 2, we will construct the evaluation index vectors; Judgment matrix and its eigenvectors will be presented in section 3; Section 4 will offer the comprehensive evaluation and some cases. Section 5 will be our conclusions.

\section{EVALUATION INDEX VECTORS}

The structure diagram for the power system communication networks is established as seen in Figure 1.

With the aid of Figure 1, we can present the index vectors:

The top level,

$$
W_{A}=\left(w_{B 1}, w_{B 2}, W_{B 3}\right)
$$

The below level,

$$
\begin{aligned}
& W_{B 1}=\left(w_{B 1 C 1}, w_{B 1 C 2}, \mathcal{W}_{B 1 C 3}, \mathcal{W}_{B 1 C 4}\right) ; \\
& W_{B 2}=\left(w_{B 2 C 5}, \mathcal{W}_{B 2 C 6}, \mathcal{W}_{B 2 C 7}\right) ; \\
& W_{B 3}=\left(w_{B 3 C 8}, w_{B 3 C 9}\right) ;
\end{aligned}
$$




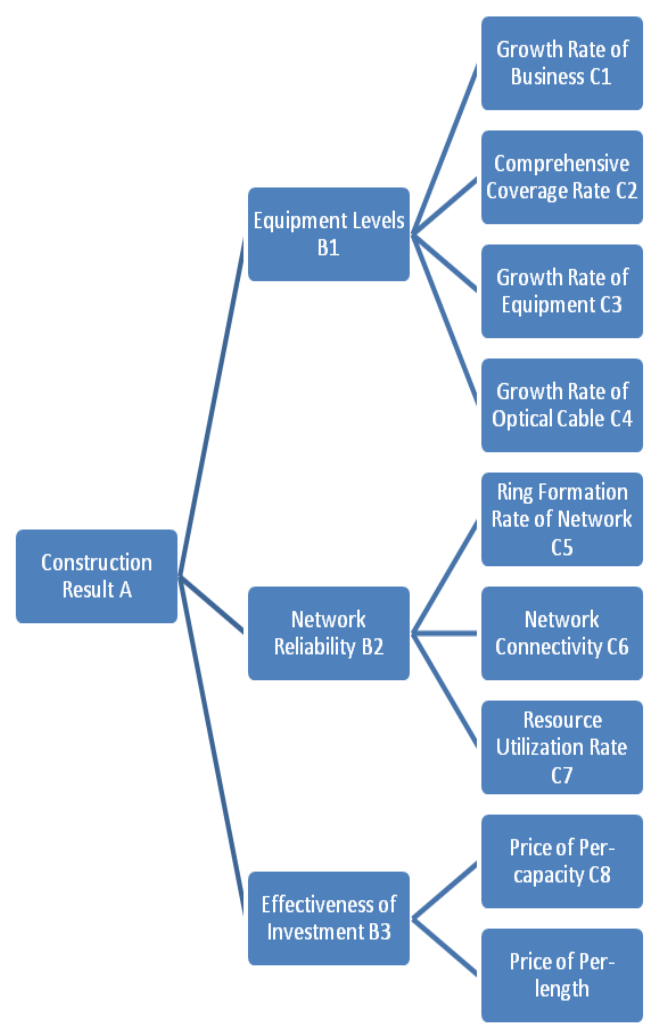

Figure 1 The structure diagram for the power system communication networks.

\section{JUDGMENT MATRIX AND ITS EIGENVCTORS}

\subsection{1-9 scales method}

To quantity the decision analysis, we compare the importance of a factor $y_{i}$ in the certain level with that of another $y_{j}(\mathrm{i}, \mathrm{j}=1, \cdots \mathrm{r})$, and illustrate that by the natural numbers $1-9$ as follows:

$L_{i j}=1$, denote that $y_{i}$ and $y_{j}$ are equally important;

$L_{i j}=3$, denote that $y_{i}$ is weakly more important than $y_{j}$;

$L_{i j}=5$, denote that $y_{i}$ is obvious more important than $y_{j}$;

$L_{i j}=7$, denote that $y_{i}$ is much more important than $y_{j}$;

$L_{i j}=9$, denote that $y_{i}$ is extremely more important than $y_{j}$;

$L_{i j}=2,4,6,8$, denote that the importance falls in between the above two degrees. Otherwise, $L_{j i}=1 / L_{i j}$.

\subsection{Judgment matrix}

According to the 1-9 scales method presented in Section 3.1, for a factor $\mathrm{x}$ in the certain level, which contains the factors $y_{k}(\mathrm{k}=1,2, \ldots \mathrm{r})$ in the next level, we can construct the judgment matrix as

$$
\left(\begin{array}{cccc}
L_{11} & L_{12} & \cdots & L_{1 r} \\
L_{21} & L_{22} & \cdots & L_{2 r} \\
\vdots & \vdots & \vdots & \vdots \\
L_{r 1} & L_{r 2} & \cdots & L_{r r}
\end{array}\right)
$$

Then the judgment matrix for the power system communication networks can be given as follows:

Tab.1 A-B Matrix

\begin{tabular}{|c|c|c|c|}
\hline A & B1 & B2 & B3 \\
\hline B1 & 1 & $1 / 2$ & $1 / 3$ \\
\hline B2 & 2 & 1 & 1 \\
\hline B3 & 3 & 1 & 1 \\
\hline
\end{tabular}

Tab.2 B1-C Matrix

\begin{tabular}{|c|c|c|c|c|}
\hline $\mathrm{B} 1$ & $\mathrm{C} 1$ & $\mathrm{C} 2$ & $\mathrm{C} 3$ & $\mathrm{C} 4$ \\
\hline $\mathrm{C} 1$ & 1 & $1 / 3$ & $1 / 2$ & $1 / 4$ \\
\hline $\mathrm{C} 2$ & 3 & 1 & 2 & 1 \\
\hline $\mathrm{C} 3$ & 2 & $1 / 2$ & 1 & 2 \\
\hline $\mathrm{C} 4$ & 4 & 1 & $1 / 2$ & 1 \\
\hline
\end{tabular}

Tab.3 B2-C Matrix

\begin{tabular}{|c|c|c|c|}
\hline B2 & C5 & C6 & C7 \\
\hline C5 & 1 & $1 / 4$ & $1 / 3$ \\
\hline C6 & 4 & 1 & 2 \\
\hline C7 & 3 & $1 / 2$ & 1 \\
\hline
\end{tabular}

Tab.4 B3-C Matrix

\begin{tabular}{|c|c|c|}
\hline B3 & C8 & C9 \\
\hline C8 & 1 & 2 \\
\hline C 9 & $1 / 2$ & 1 \\
\hline
\end{tabular}

\subsection{Eigenvectors}

In this section, we will derive the eigenvectors of the judgment matrix for the power system communication networks as

$$
\begin{aligned}
W_{A} & =\left(w_{B 1}, w_{B 2}, w_{B 3}\right) \\
= & (0.1570,0.3494,0.5936) ; \\
W_{B 1} & =\left(\begin{array}{lll}
w_{B 1 C 1}, & w_{B 1 C 2}, & w_{B 1 C 3}, W_{B 1 C 4}
\end{array}\right) \\
& =(0.1028,0.3560,0.2706,0.2760) ; \\
W_{B 2} & =\left(\begin{array}{lll}
w_{B 2 C 5}, w_{B 2 C 6}, w_{B 2 C 7}
\end{array}\right) \\
& =(0.1320,0.5584,0.3196) ; \\
W_{B 3} & =\left(w_{B 3 C 8}, w_{B 3 C 9}\right)=(0.2000,0.1000) ;
\end{aligned}
$$




\section{COMPREHENSIVE EVALUATION}

\subsection{Comprehensive index vector}

The evaluation index vector for the construction results of the power system communication networks $\mathrm{P}$ can be obtained through some calculations as

$$
\begin{aligned}
& \mathcal{W}_{1}=\mathcal{W}_{B 1} \times \mathcal{W}_{B 1 C 1}=0.1570 \times 0.1028=0.0161, \\
& \mathcal{W}_{2}=\mathcal{W}_{B 1} \times \mathcal{W}_{B 1 C 2}=0.1570 \times 0.3560=0.0559 \\
& \mathcal{W}_{3}=\mathcal{W}_{B 1} \times \mathcal{W}_{B 1 C 3}=0.1570 \times 0.2706=0.0425 \\
& \mathcal{W}_{4}=\mathcal{W}_{B 1} \times \mathcal{W}_{B 1 C 4}=0.1570 \times 0.2760=0.0433 \\
& \mathcal{W}_{5}=\mathcal{W}_{B 2} \times \mathcal{W}_{B 2 C 5}=0.3494 \times 0.1320=0.0461, \\
& \mathcal{W}_{6}=\mathcal{W}_{B 2} \times \mathcal{W}_{B 2 C 6}=0.3494 \times 0.5584=0.1951, \\
& \mathcal{W}_{7}=\mathcal{W}_{B 2} \times \mathcal{W}_{B 2 C 7}=0.3494 \times 0.3196=0.1117 \\
& \mathcal{W}_{8}=\mathcal{W}_{B 3} \times \mathcal{W}_{B 3 C 8}=0.5936 \times 0.2000=0.1187 \\
& \mathcal{W}_{9}=\mathcal{W}_{B 3} \times \mathcal{W}_{B 3 C 9}=0.5936 \times 0.1000=0.0594
\end{aligned}
$$

and that

$$
\begin{aligned}
& \mathrm{P}=\left(w_{1}, w_{2}, w_{3}, w_{4}, w_{5}, w_{6}, w_{7}, w_{8}, w_{9}\right) \\
& =(0.0161,0.0559,0.0425,0.0433,0.0461,0.1951,0.1117,0.11 \\
& 87,0.0594)
\end{aligned}
$$

\subsection{Established case}

Firstly, rating system of the vector element is designed according to the Fuzzy Decision Theory, as follows:

Tab.5 Rating system of vector element.

\begin{tabular}{|c|c|c|c|c|c|c|c|c|c|c|}
\hline $\mathrm{Si}$ & 100 & 90 & 80 & 70 & 60 & 50 & 40 & 30 & 20 & 10 \\
\hline $\mathrm{N}$ & $\mathrm{n} 1$ & $\mathrm{n} 2$ & $\mathrm{n} 3$ & $\mathrm{n} 4$ & $\mathrm{n} 5$ & $\mathrm{n} 6$ & $\mathrm{n} 7$ & $\mathrm{n} 8$ & $\mathrm{n} 9$ & $\mathrm{n} 10$ \\
\hline
\end{tabular}

where $\sum_{i=1}^{10} n_{i}=N$

Then the final score can be obtained by

$$
\text { Score }=\sum_{i=1}^{10} n_{i} S i / N \text {. }
$$

For example, to the $\mathrm{Cj}=[0.08,0.09]$ and $\mathrm{N}=30$,

Tab.6 Case of the rating system.

\begin{tabular}{|c|c|c|c|c|c|c|c|c|c|c|}
\hline $\mathrm{Si}$ & 100 & 90 & 80 & 70 & 60 & 50 & 40 & 30 & 20 & 10 \\
\hline $\mathrm{N}$ & 3 & 6 & 6 & 7 & 5 & 2 & 1 & 0 & 0 & 0 \\
\hline
\end{tabular}

Then the score for $\mathrm{Cj}$ is 75 .

Based on above designed formula, each element of index vector can be graded with the centesimal as
Tab.7 Scores of the element of index vector $\mathrm{Cj}$.

\begin{tabular}{|c|c|}
\hline $\mathrm{Cj}$ & score \\
\hline$[0.1,1]$ & 100 \\
\hline$[0.09,0.1]$ & 80 \\
\hline$[0.08,0.09]$ & 75 \\
\hline$[0.07,0.08]$ & 70 \\
\hline$[0.06,0.07]$ & 65 \\
\hline$[0.05,0.06]$ & 60 \\
\hline$[0.04,0.05]$ & 55 \\
\hline$[0.03,0.04]$ & 50 \\
\hline$[0,0.03]$ & 30 \\
\hline
\end{tabular}

Then we will take $\mathrm{K} 1, \mathrm{~K} 2$, $\mathrm{K} 3$ and $\mathrm{K} 4$ provinces as examples,

Tab.8 Case of the comprehensive evaluation for construction results of the power system communication networks.

\begin{tabular}{|c|c|c|c|c|}
\hline & $\mathrm{K} 1$ & $\mathrm{~K} 2$ & $\mathrm{~K} 3$ & $\mathrm{~K} 4$ \\
\hline $\mathrm{C} 1$ & 0.06 & 0.05 & 0.07 & 0.06 \\
\hline $\mathrm{C} 2$ & 0.05 & 0.07 & 0.05 & 0.06 \\
\hline $\mathrm{C} 3$ & 0.1 & 0.08 & 0.06 & 0.05 \\
\hline $\mathrm{C} 4$ & 0.04 & 0.04 & 0.05 & 0.07 \\
\hline $\mathrm{C} 5$ & 0.07 & 0.06 & 0.07 & 0.05 \\
\hline C6 & 0.02 & 0.02 & 0.03 & 0.05 \\
\hline C7 & 0.06 & 0.05 & 0.06 & 0.06 \\
\hline C8 & 0.07 & 0.05 & 0.05 & 0.07 \\
\hline C 9 & 0.08 & 0.05 & 0.06 & 0.05 \\
\hline
\end{tabular}

\subsection{Synthesize}

On the basis of Sections 4.1 and 4.2, the 9 factors in the comprehensive index vector can be synthesized into one as

$$
\begin{aligned}
W_{K 1} & =P(65,60,100,50,70,30,65,70,70)^{T} \\
& =39.623 ; \\
W_{K 2} & =P(60,65,75,50,65,30,60,60,60)^{T} \\
& =36.190 ; \\
W_{K 3} & =P(70,60,65,60,70,50,65,60,65)^{T} \\
& =41.067 \\
W_{K 4} & =P(65,65,60,70,60,60,65,70,60)^{T} \\
& =44.164 .
\end{aligned}
$$

From the above calculations, we can see that construction results of the power system communication networks in the areas $\mathrm{K} 1, \mathrm{~K} 2, \mathrm{~K} 3$ and $\mathrm{K} 4: W_{K 4} \geq W_{K 3} \geq W_{K 1} \geq W_{K 2}$. 


\section{CONCLUSIONS}

With the Analytic Hierarchy Process (AHP) and Fuzzy Decision Theory (FDT), we have investigated the comprehensive evaluation for construction results of the power system communication networks, which can be used to provide necessary basis for decision making with certain credibility. The area $\mathrm{K} 1, \mathrm{~K} 2, \mathrm{~K} 3$ and $\mathrm{K} 4$ have been taken as examples, through which we have found that construction results of the power system communication networks of $\mathrm{K} 1, \mathrm{~K} 2, \mathrm{~K} 3$ and $\mathrm{K} 4$ : $W_{K 4} \geq W_{K 3} \geq W_{K 1} \geq W_{K 2}$. This work may be useful in studying the construction results of the power system communication networks.

\section{REFERENCES}

[1] Ding Kai-sheng \& Liang Xiong-jian 1998 Reliability Management and Comprehensive Evaluation Method on Telecommunication Networks.

[2] Li Wei-guo et al. 2012 Application of fuzzy analytic hierarchy process and neural network in power transformer risk assessment.

[3] Zhu Jian-jun (2005) Research on Some Problems of the Analytic Hierarchy Process and Its Application.

[4] Han Yang \& Dai Fa-shan 2012 The Research on the Location Selection of the Bank Outlets Based on Triangular Fuzzy Analytic Hierarchy Process.

[5] Wang Xin-biao et al. 2012 Application of Fuzzy Mathematics Theory to Set up Sports and Health Course of Study Evaluating System. 